\title{
Relações entre Conjugalidade dos Pais, Conjugalidade dos Filhos e Bem-Estar Subjetivo
}

\author{
Fabio Scorsolini-Comin - Universidade Federal do Triângulo Mineiro, Uberaba, Brasil \\ Anne Marie Germaine Victorine Fontaine - Universidade do Porto, Porto, Portugal \\ Sabrina Martins Barroso - Universidade Federal do Triangulo Mineiro, Uberaba, Brasil \\ Manoel Antônio dos Santos - Universidade de São Paulo, Ribeirão Preto, Brasil
}

\begin{abstract}
Resumo
O objetivo deste estudo foi investigar as relações existentes entre a medida da conjugalidade dos pais, tal como percebida por filhos casados, e medidas do bem-estar subjetivo e da satisfação em relacionamentos amorosos apresentados por esses filhos. Os participantes foram 118 brasileiros casados havia 16,17 ( $D P=9,98)$ anos, com média de idade de 41,55 (DP = 10,01). Os instrumentos utilizados foram: Escala de Bem-estar Subjetivo, Questionário de Conjugalidade dos Pais e Escala Fatorial de Satisfação com o Relacionamento de Casal. Foram calculadas correlações entre as medidas das variáveis contínuas dos fatores dos instrumentos pelo coeficiente de Spearman, que apontaram que a percepção dos filhos sobre a conjugalidade dos pais não está correlacionada com as demais variáveis. A satisfação no relacionamento conjugal se correlacionou satisfatoriamente com os afetos positivos, evidenciando a necessidade de considerar as transformações individuais a partir da própria experiência conjugal.

Palavras-chave: relações conjugais, casamento, bem-estar subjetivo, satisfação conjugal
\end{abstract}

\section{Relations Between Parents' Marital Relationships, Son's Marital Relationships and Subjective Well-Being}

\begin{abstract}
The aim of this study was to investigate the relations between the measure of marital relationships of parents, as perceived by married children, and measures of subjective well-being and satisfaction in romantic relationships. There were 118 Brazilian married individuals $(M=16.17$ years; $S D=9.98)$, with a mean age of $41.55(S D=10.01)$. The instruments used were: Scale of Subjective Well-being, Conjugality Parental Questionnaire and Scale Factor Satisfaction with Couple Relationships. We calculated the correlations between measurements of continuous variables of the factors of the instruments by Spearman coefficient, which indicated that the perception of sons and daughters on the parents' marital is not correlated with other variables. The marital relationship satisfaction was correlated with positive affect, highlighting the need to close readings of individual transformations from one's own marital experience.

Keywords: marital relationships, marriage, subjective well-being, marital satisfaction
\end{abstract}

\section{Relaciones entre la Conyugalidad de los Padres, Conyugalidad de los Hijos y Bienestar Subjetivo}

\section{Resumen}

El objetivo de este estudio fue investigar la relación existente entre la medida de conyugalidad de los padres, según la percepción de los hijos casados, y medidas de bienestar subjetivo y de satisfacción en las relaciones amorosas presentadas por esos hijos. Los participantes fueron 118 brasileños casados hacía 16,17 $(D P=9,98)$ años, y edad media de 41,55 $(D P=10,01)$. Los instrumentos utilizados fueron: Escala de Bienestar Subjetivo, Cuestionario de Conyugalidad de los padres y Escala Factorial de Satisfacción con el relacionamiento de la pareja. Se calcularon correlaciones entre las medidas de las variables continuas de los factores de los instrumentos, por medio del coeficiente de Spearman, que señalaron que la percepción de los hijos sobre el matrimonio de los padres no está relacionada con las demás variables. La satisfacción en el relacionamiento marital se correlacionó satisfactoriamente con los afectos positivos, dejando en evidencia la necesidad de considerar las transformaciones individuales a partir de la propia experiencia marital.

Palabras clave: relaciones conjugales, matrimonio, bienestar subjetivo, satisfacción conjugal

A despeito de certos discursos contemporâneos que valoram o casamento como um acontecimento negativo ou fadado ao fracasso, dados recentes da pesquisa anual de Estatísticas de Registro Civil do Instituto Brasileiro de Geografia e Estatística (IBGE, 2011) revelaram que a taxa de casamentos no Brasil vem crescendo a cada ano, especialmente desde 2003. Em 2010, foram registrados 977.620 casamentos, com aumento de $4,5 \%$ em relação ao ano anterior. A maior parte desses casamentos envolveu cônjuges solteiros $(81,7 \%)$.
A conjugalidade pode ser definida como dimensão psicológica compartilhada, com uma dinâmica inconsciente regulada por leis e funcionamento específicos. É um processo de construção de uma realidade comum (Féres-Carneiro, Ziviani, \& Magalhães, 2007). Nessa concepção, a conjugalidade é constituída por um sistema com funcionamento autônomo, sujeito a conflitos ou crises que remetem à dinâmica inconsciente compartilhada. A vinculação na díade é importante para a intimidade e para o investimento na relação amorosa, 
o que está associado à satisfação conjugal. A intimidade é uma experiência que tem início nas primeiras relações estabelecidas na infância e, como tal, ultrapassa a noção de proximidade afetiva. Contempla um conjunto de percursos desenvolvimentais e implica em movimentos constantes de autonomia e diferenciação.

Existem várias perspectivas de análise do modo como as experiências vivenciadas na família de origem estão relacionadas com as formas com que os filhos irão negociar e desenvolver seus relacionamentos íntimos no futuro, incluindo representações de desenvolvimento e expectativas de si mesmo e de outros relacionamentos, por meio de uma repetição direta dos processos e dinâmicas emocionais de suas família de origem (Bowlby, 1969/1990; Hare, Miga, \& Allen, 2009). Tal influência passaria também pela construção da identidade dos filhos por meio dos estilos parentais vivenciados, sobretudo na infância e na adolescência (Marin, Piccinini, \& Tudge, 2011; Moreira, Rabinovich, \& Silva, 2009; Seligman, 2011; Snyder \& Lopez, 2009).

O pressuposto de que a família de origem é fundamental para a transmissão não apenas de valores, mas de posturas e formas de se vivenciar o afeto, é corroborado por uma gama expressiva de estudos recentes (David, Gelberg, \& Suchman, 2012; Lau, Beilby, Byrnes, \& Hennessey, 2012; Vieira, Ávila, \& Matos, 2012). Muitos estudos internacionais também têm destacado que os comportamentos conjugais de pais e mães desempenham um papel central nas atitudes de adolescentes e jovens adultos em relação ao casamento, o que se acentua quando se iniciam as primeiras relações amorosas (Willoughby, Carroll, Vitas, \& Hill, 2012). Se a conjugalidade dos pais está diretamente relacionada à conjugalidade dos filhos, Seligman (2011) afirma que as experiências amorosas positivas na vida adulta (por exemplo, o casamento) estariam na base do conceito de bem-estar.

A partir do exposto, o objetivo deste estudo foi investigar as relações existentes entre a medida da conjugalidade dos pais, tal como percebida por filhos casados, e as medidas do bem-estar subjetivo e da satisfação em relacionamentos amorosos apresentados por esses filhos. As hipóteses delineadas foram: H1 - A medida da percepção que os filhos têm acerca do relacionamento conjugal de seus pais está positivamente correlacionada à medida da percepção acerca de aspectos específicos de seus próprios relacionamentos; $\mathrm{H} 2$ - A medida da percepção que os filhos têm acerca de aspectos específicos de seus próprios relacionamentos está positivamente correlacionada com a medida de seus níveis de bem-estar subjetivo; H3 -A medida do bem-estar subjetivo dos filhos está correlacionada com a medida da satisfação global percebida no relacionamento conjugal dos pais.

\section{Método}

Trata-se de um estudo descritivo e correlacional, de corte transversal, de caráter exploratório e apoiado na metodologia quantitativa de análise e interpretação dos dados.

\section{Participantes}

O estudo contou com uma amostra de pessoas de ambos os sexos, casadas consensualmente em primeiras núpcias com, no mínimo, dois anos, e que obedecessem aos seguintes critérios: (a) heterossexuais; (b) não tivessem se separado ou não estivessem em processo de divórcio; (c) com ou sem filhos; (d) com pais igualmente casados em primeiras núpcias; (e) grau de instrução médio-superior; (f) sem indícios aparentes ou relatados de distúrbios comportamentais ou déficit cognitivo; (g) sem restrição de idade máxima. Essa não restrição de idade máxima para participar deveu-se ao aumento da idade com que as pessoas têm se casado no Brasil, ou seja, há menos pessoas casadas na população de 18 a 30 anos do que nos estratos etários superiores, segundo dados brasileiros oficiais (IBGE, 2011).

A saturação da amostra foi definida por meio dos procedimentos preconizados por Krejcie e Morgan (1970). O N da população foi estimado a partir dos dados oficiais do IBGE de pessoas casadas e solteiras na população por faixa etária e grau de escolaridade. Os participantes foram localizados em uma cidade de porte médio localizada no interior do Estado de São Paulo, Brasil. Foi realizado o cálculo de poder estatístico da amostra considerando o modelo de Schlesselman (Moraes \& Souza, 1998). Após a aplicação do modelo, concluiu-se que o poder da amostra é de $73 \%$, que é considerado um valor aceitável. Sendo assim, a amostra foi do tipo não probabilística, composta por critérios de conveniência, totalizando 118 participantes. As características da amostra foram sumarizadas na Tabela 1.

\section{Instrumentos}

(a) Questionário de Identificação do Participante (Scorsolini-Comin \& Santos, 2012): questionário desenvolvido com o objetivo de fornecer dados gerais do participante, como idade, sexo, grau de instrução, atividade profissional, renda familiar, 
Tabela 1

Caracterização Sociodemográfica da Amostra $(N=118)$

\begin{tabular}{|c|c|c|}
\hline Variáveis & $\mathrm{n}(\%)$ & Média (DP) \\
\hline \multicolumn{3}{|l|}{ Sexo } \\
\hline Masculino & $33(27,96)$ & \\
\hline Feminino & $85(72,03)$ & \\
\hline Idade (em anos) & & $41,55(10,01)$ \\
\hline Renda familiar (em reais) & & $5.172,17(4.184,50)$ \\
\hline \multicolumn{3}{|l|}{ Classificação socioeconômica } \\
\hline Classe A & $14(11,86)$ & \\
\hline Classe B & $89(75,42)$ & \\
\hline Classe C & $15(12,71)$ & \\
\hline Classe D & - & \\
\hline Classe E & - & \\
\hline \multicolumn{3}{|l|}{ Grau de instrução } \\
\hline Ensino Fundamental Incompleto & $4(3,38)$ & \\
\hline Ensino Fundamental Completo & $3(2,54)$ & \\
\hline Ensino Médio Incompleto & $2(1,69)$ & \\
\hline Ensino Médio Completo & $18(15,25)$ & \\
\hline Ensino Superior Incompleto & $11(9,32)$ & \\
\hline Ensino Superior Completo & $80(67,79)$ & \\
\hline \multicolumn{3}{|l|}{ Profissão/Ocupação } \\
\hline Professor/Ensino Fundamental & $33(27,96)$ & \\
\hline Professor/Ensino Superior & $12(10,16)$ & \\
\hline Psicólogo & $6(5,08)$ & \\
\hline Do lar & $6(5,08)$ & \\
\hline Outras & $61(51,69)$ & \\
\hline Tempo de casamento (em anos) & & $16,17(9,98)$ \\
\hline Grau de satisfação no casamento & & $4(0,89)$ \\
\hline Nem um pouco & $2(1,69)$ & \\
\hline Um pouco & $4(3,38)$ & \\
\hline Moderadamente satisfatório & $23(19,49)$ & \\
\hline Bastante satisfatório & $52(44,06)$ & \\
\hline Extremamente satisfatório & $37(31,35)$ & \\
\hline \multicolumn{3}{|l|}{ Chefe de família } \\
\hline Próprio participante & $50(42,37)$ & \\
\hline Companheiro, esposo, namorado & $61(51,69)$ & \\
\hline Companheira, esposa, namorada & $7(5,93)$ & \\
\hline \multicolumn{3}{|l|}{ Reside com os pais } \\
\hline $\operatorname{Sim}$ & $6(5,08)$ & \\
\hline Não & $108(91,52)$ & \\
\hline Sem resposta & $4(3,38)$ & \\
\hline \multicolumn{3}{|l|}{ Idade dos pais (em anos) } \\
\hline Pais & & $69,24(11,61)$ \\
\hline Mães & & $66,54(11,89)$ \\
\hline Tempo de casamento dos pais (em anos) & & $40,44(10,90)$ \\
\hline
\end{tabular}


tempo de duração do relacionamento e se tem filhos ou não. Nesse instrumento, foi incluída uma pergunta, aqui identificada como Satisfação Global com o Relacionamento Conjugal (SR), disposta em uma escala Likert que ia de 1 - Nem um ponco satisfatório a 5-Extremamente satisfatório.

(b) Classificação Socioeconômica: o critério Abipeme (2008) é uma escala de classificação socioeconômica por intermédio da atribuição de pesos a um conjunto de itens de conforto doméstico, além do nível de escolaridade do chefe de família. A classificação socioeconômica da população é estratificada em cinco classes, denominadas A, B, C, D e E, que correspondem à pontuação obtida pelo participante.

\section{(c) Escala de Bem-estar Subjetivo - EBES} (Albuquerque \& Tróccoli, 2004): trata-se de um instrumento inspirado em escalas extraídas da literatura internacional. A análise fatorial dos componentes principais e a análise fatorial (extração dos eixos principais - PAF e rotação Oblimin) revelaram três fatores: afeto positivo (21 itens, explicando $24,3 \%$ da variância, $a=0,95)$; afeto negativo (26 itens, $24,9 \%$ da variância, $a=0,95)$ e satisfação-insatisfação com a vida (15 itens, $21,9 \%$ da variância, $a=0,90)$. Juntos, os três fatores explicaram $44,1 \%$ da variância total do construto (Albuquerque \& Tróccoli, 2004). Na presente amostra $(N=118)$, os alfas de Cronbach encontrados foram: afetos positivos $(a=0,90)$, afetos negativos ( $a$ $=0,94)$ e satisfação com a vida $(a=0,90)$, considerados satisfatórios (Pasquali, 2001). O instrumento é composto por duas partes com subescalas. A primeira parte agrupa as subescalas dos afetos positivos e negativos, e a segunda a satisfação com a vida. $\mathrm{Na}$ primeira parte, os itens variam do número 1 ao 47 e descrevem afetos positivos e negativos, devendo o sujeito responder como tem se sentido ultimamente em uma escala Likert, na qual 1 significa "nem um pouco" e 5 significa "extremamente". Na segunda parte, os itens vão do número 48 ao 62 e descrevem julgamentos relativos à avaliação de satisfação ou insatisfação com a vida, devendo ser respondidos em uma escala Likert na qual 1 significa "discordo plenamente" e 5 significa "concordo plenamente".

(d) Questionário de Conjugalidade dos Pais QCP (Féres-Carneiro, Ziviani, \& Magalhães, 2007). A versão do QCP aplicada neste estudo é constituída por 56 itens, a serem respondidos em escala Likert de cinco pontos, sendo que "nunca" corresponde a 1 e "sempre" corresponde a 5. O instrumento permite que se utilizem as características psicométricas que sugerem a unidimensionalidade da escala de avaliação do QCP, dirigida ao construto maior: a conjugalidade dos pais percebida pelos filhos (Ziviani, Féres-Carneiro, \& Magalhães, 2011). No estudo original, o alfa de Cronbach encontrado foi de 0,96 . Na presente amostra, o índice obtido também foi de 0,96 , considerado satisfatório.

(e) Escala Fatorial de Satisfação com o Relacionamento de Casal - EFS-RC (Wachelke, Andrade, Souza, \& Cruz, 2007): instrumento autoadministrado breve, desenvolvido e validado no contexto brasileiro. Composta por nove itens do tipo Likert, é subdividida em duas dimensões de avaliação de esferas específicas do relacionamento de casal: satisfação com a atração física e sexualidade (SAFS) e satisfação em relação às afinidades de interesses e comportamentos (SAIC) entre companheiros de relação. $\mathrm{O}$ alfa de Cronbach calculado para o SAFS foi de 0,80 , e para o SAIC de 0,68 (Wachelke et al., 2007). O alfa de Cronbach calculado para a medida de satisfação global foi de $0,90 . \mathrm{Na}$ presente amostra, foram obtidos os seguintes índices: SAFS $(a=0,77)$, SAIC $(a=0,64)$, escala total $(a=0,79)$.

\section{Procedimento}

Coleta de Dados. Todos os participantes foram contatados pessoalmente pelo pesquisador e a aplicação dos instrumentos foi realizada individualmente. Mesmo em se tratando de casais, cada membro respondeu de modo independente (sem a presença do cônjuge). Outro cuidado para assegurar a aplicação independente foi de que os respondentes não tivessem acesso às respostas do parceiro nem conversado com ele após a aplicação. Os participantes foram contatados por meio do procedimento "bola de neve", em que novos sujeitos foram indicados pelos próprios respondentes, que tinham sido contatados previamente pelo pesquisador.

\section{Análise dos Dados}

Após aplicação dos instrumentos, foi montado um banco de dados na planilha Excel, posteriormente transposto para programa estatístico para operacionalização das análises. Para garantir que todas as respostas fossem transpostas corretamente, utilizou-se a técnica da dupla digitação por dois pesquisadores diferentes, sendo um deles o responsável pela pesquisa. Os dados obtidos com a aplicação dos instrumentos foram transpostos para o Software SPSS for Windows versão 17.0. Primeiramente, foram feitas as análises descritivas para caracterização da amostra (médias, medianas, desvios-padrão e porcentagens). Foi aplicado o teste de normalidade Kolmogorov-Smirnov e a análise 
de resíduos. Como os dados não mostraram seguir distribuição normal, foi empregada estatística não paramétrica para análise de correlações. Foram calculadas as correlações entre as medidas das variáveis contínuas dos fatores dos instrumentos apresentados. A correlação dos fatores dos três instrumentos foi dada a partir do coeficiente de correlação de Spearman. A força da grandeza do coeficiente de correlação entre as variáveis foi avaliada conforme procedimento proposto por Ajzen e Fishbein (1998), que consideram os valores de correlação próximos de 0,30 satisfatórios, entre $0,30 \mathrm{e}$ 0,50 como de moderada magnitude e acima de 0,50 de forte magnitude. Valores abaixo de 0,30 são de pouco valor para a prática, mesmo que estatisticamente significante. $O$ nível de significância adotado no exame das correlações foi $p \leq 0,05$.

\section{Considerações Éticas}

Este estudo foi aprovado pelo Comitê de Ética em Pesquisa da instituição de origem do primeiro autor (Processo 439/2009).

\section{Resultados}

No que concerne aos fatores do bem-estar subjetivo (BES), os resultados mostram que os afetos positivos (AP) estão negativamente correlacionados com os afetos negativos (AN) (rho $=-0,24 ; p=0,007$ ) e positivamente com a satisfação com a vida (SV) (rho $=0,23 ; p=0,10)$, seguindo a tendência do estudo de validação da Escala de Bem-estar Subjetivo, que contou com a participação de pessoas solteiras e casadas (Albuquerque \& Tróccoli, 2004) e de um estudo realizado apenas com pessoas casadas (Scorsolini-Comin \& Santos, 2012). Na amostra do presente estudo, no entanto, as correlações encontradas foram consideradas fracas (respondem por menos de $6 \%$ da variância comum).

Os AP também estão moderadamente correlacionados com a satisfação com a atração física e sexualidade, SAFS ( $r$ ro $=0,44 ; p \leq 0,001)$, mas não de modo significativo com a satisfação com afinidades de interesse e comportamentos, SAIC. Adicionalmente, os AP se correlacionam fortemente com o grau de satisfação com o relacionamento ( $r h o=0,50 ; p \leq 0,001)$. Os AP não estão significativamente associados com a percepção dos filhos sobre a conjugalidade dos pais.

Os AN estão negativa e moderadamente correlacionados com a SAFS $(r h o=-0,27 ; p=0,002)$ e com o grau de satisfação com o relacionamento atual (rho $=-0,27 ; p=0,02)$. A partir desses achados, podemos inferir que pessoas mais propensas à depressão tenderiam a avaliar de modo mais negativo não apenas seus relacionamentos amorosos (no caso desse grupo, o casamento) como os dos seus pais, o que nos leva a considerar a influência de um componente interno e individual (afetos negativos) na avaliação dos relacionamentos com outras pessoas, tanto em termos da parceria conjugal como do casal parental.

A satisfação com a vida, SV, terceiro fator do $\mathrm{BES}$, só se correlacionou com os AP, não apresentando qualquer associação com a percepção de conjugalidade dos pais e de conjugalidade do casal. Em estudos anteriores (Scorsolini-Comin \& Santos, 2010, 2012), constatou-se que tal domínio estava positiva e significativamente correlacionado como a satisfação diádica em pessoas casadas.

Em termos dos fatores da satisfação conjugal, a SAFS está moderadamente correlacionada com os AP $(r h o=0,44 ; p \leq 0,001), \mathrm{AN}(r h o=-0,27 ; p=0,002), \mathrm{e}$ fortemente com o grau de satisfação com o relacionamento conjugal, SR $(r h o=0,58 ; p \leq 0,001)$. Foi visto que SAIC não se correlacionou significativamente com os AP, os AN, com a SAFS, com a percepção sobre a conjugalidade dos pais, mas se correlacionou moderadamente com o grau de satisfação com o relacionamento conjugal $(r h o=0,30 ; p=0,01)$.

A percepção dos filhos sobre a conjugalidade dos pais (PCP), principal variável deste estudo, embora esteja significativamente correlacionada aos AP e à SAIC, apresenta uma baixa força de grandeza nessas associações, com pouca capacidade de explicação do fenômeno. Essa baixa magnitude não nos permite afirmar, inequivocamente, que a conjugalidade dos pais esteja correlacionada com a conjugalidade dos filhos, devendo esse achado ser analisado com parcimônia. Essas correlações da PCP com as demais variáveis estão representadas na Figura 1.

O grau de satisfação com o relacionamento, no caso, o próprio casamento, está significativamente correlacionado com os AP ( $r h o=0,50 ; p \leq 0,001)$, SAFS $(r b o=0,58 ; p \leq 0,001)$ e moderadamente com os AN $(r h o=-0,27 ; p=0,002)$ e SAIC $(r h o=0,30 ; p=0,001)$, comprovando a tendência expressa na amostra total de que pessoas satisfeitas com seus relacionamentos em termos mais gerais também o seriam em termos de comportamentos, afinidades e sexualidade, e manifestariam mais aspectos positivos e menos aspectos negativos. Essas correlações estão representadas na Tabela 2. A Figura 2 representa, de modo sintético, as correlações mais significativas (moderadas e fortes). 


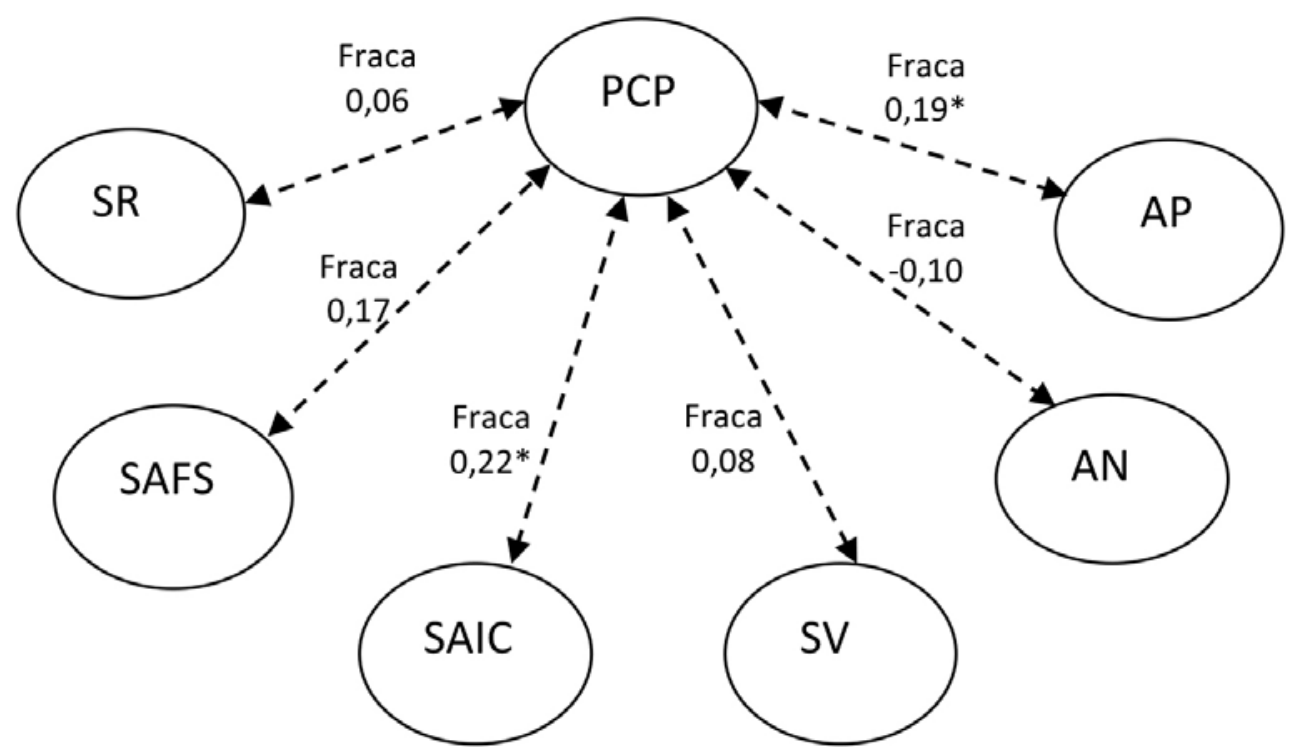

Figura 1. Representação das correlações obtidas entre a percepção dos filhos sobre a conjugalidade dos pais (PCP) e as variáveis de satisfação no relacionamento (SR, SAFS, SAIC) e bem-estar subjetivo (AP, AN, SV) (N = 118).

${ }^{*} p \leq 0,05 * * \leq 0,01$.

Nota. $\mathrm{AP}=$ afetos positivos; $\mathrm{AN}=$ afetos negativos; $\mathrm{SV}=$ satisfação com a vida; $\mathrm{SR}=$ satisfação no relacionamento; $\mathrm{SAFS}=$ satisfação com a atração física e sexualidade; SAIC = satisfação com a afinidade de interesses e comportamentos; linhas tracejadas representam as correlações não significativas.

Tabela 2

Correlações e Nivel de Significância Entre os Fatores de Bem-estar Subjetivo, Satisfação em Relacionamento de Casal e Percepção dos Filhos Sobre a Conjugalidade dos Pais em Pessoas Casadas ( $N=118)$

\begin{tabular}{|c|c|c|c|c|c|c|c|}
\hline & AP & AN & SV & SAFS & SAIC & PCP & SR \\
\hline \multirow[t]{2}{*}{ AP } & - & $-0,24 * *$ & $0,23^{*}$ & $0,44 * *$ & $0,20 *$ & $0,19 *$ & $0,50 * *$ \\
\hline & & 0,07 & 0,10 & 0,00 & 0,24 & 0,03 & 0,00 \\
\hline \multirow[t]{2}{*}{ AN } & & - & $-0,076$ & $-0,27 * *$ & $-0,18^{*}$ & $-0,10$ & $-0,27 * *$ \\
\hline & & & 0,41 & 0,00 & 0,04 & 0,28 & 0,00 \\
\hline \multirow[t]{2}{*}{ SV } & & & - & 0,11 & 0,13 & 0,08 & 0,070 \\
\hline & & & & 0,20 & 0,14 & 0,36 & 0,45 \\
\hline \multirow[t]{2}{*}{ SAFS } & & & & - & $0,23 * *$ & 0,17 & $0,58^{* *}$ \\
\hline & & & & & 0,00 & 0,06 & 0,00 \\
\hline \multirow[t]{2}{*}{ SAIC } & & & & & - & $0,22 *$ & $0,30 * *$ \\
\hline & & & & & & 0,17 & 0,00 \\
\hline \multirow[t]{2}{*}{ РCP } & & & & & & - & 0,06 \\
\hline & & & & & & & 0,51 \\
\hline SR & & & & & & & - \\
\hline
\end{tabular}

${ }^{*} p \leq 0,05 ; * * \leq 0,01$

Nota. $\mathrm{AP}=$ afetos positivos; $\mathrm{AN}=$ afetos negativos; $\mathrm{SV}=$ satisfação com a vida; $\mathrm{SAFS}=$ satisfação com a atração física e sexualidade; $\mathrm{SAIC}=$ satisfação com a afinidade de interesses e comportamentos; PCP = percepção dos filhos sobre a conjugalidade dos pais; SR = grau de satisfação com o relacionamento. 


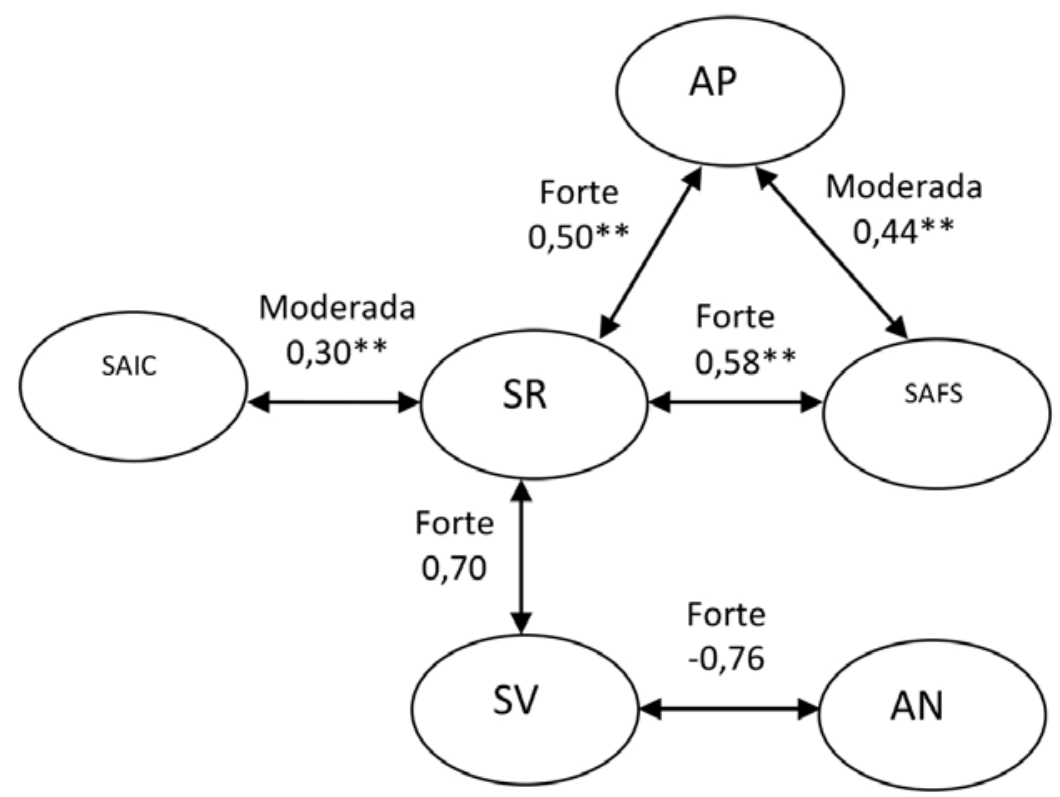

Figura 2. Representação das principais correlações (moderadas e fortes) obtidas entre os fatores de bem-estar subjetivo, satisfação em relacionamento de casal e percepção dos filhos sobre a conjugalidade dos pais em pessoas casadas $(N=118) .{ }^{*} p \leq 0,05$; ${ }^{* *} p$ $\leq 0,01$.

Nota. $\mathrm{AP}=$ afetos positivos; $\mathrm{AN}=$ afetos negativos; $\mathrm{SV}=$ satisfação com a vida; $\mathrm{SR}=$ satisfação no relacionamento; $\mathrm{SAFS}=$ satisfação com a atração física e sexualidade; SAIC = satisfação com a afinidade de interesses e comportamentos.

Retornando às hipóteses norteadoras deste estudo, a primeira hipótese, segundo a qual a percepção dos filhos sobre a conjugalidade dos pais seria correlacionada com a satisfação no relacionamento atual, foi parcialmente comprovada, uma vez que a PCP se correlacionou fracamente com a SAIC $(r h o=0,22 ; p$ $=0,017)$. A segunda hipótese, de que a satisfação no relacionamento conjugal atual estaria correlacionada com o nível de bem-estar subjetivo, foi parcialmente confirmada, uma vez que apenas os AP e os AN estão correlacionados com os fatores SAFS, SAIC e SR. $\mathrm{O}$ terceiro fator do BES, a SV, não se correlacionou significativamente com qualquer fator de satisfação conjugal. A terceira hipótese delineada, de que o BES estaria correlacionado à PCP, foi também parcialmente comprovada, uma vez que apenas os AP mostraram-se correlacionados à PCP. Essa associação, no entanto, é de baixa magnitude e com pouco poder de explicação do fenômeno.

\section{Discussão}

No que concerne aos fatores do BES, confirma-se a robustez do instrumento EBES para a avaliação dessa amostra, o que se verificou também em outra amostra de pessoas casadas, investigada em estudo anterior (Scorsolini-Comin \& Santos, 2012). Assim, os AP, AN e SV estão correlacionados na amostra de casados do presente estudo. O nível de AP também está significativamente correlacionado com a SAFS $(r b o=$ $0,44 ; p \leq 0,001)$ e com o fator unidimensional da satisfação em relacionamento (SR) $(r h o=0,50 ; p \leq 0,001)$. Assim, quanto maior o nível de bem-estar da pessoa casada, maior a sua satisfação com o seu casamento. Quanto menor o seu bem-estar, ou seja, quanto mais experimenta sentimentos como desconsolo, tristeza, desamparo e depressão, pior é a avaliação que faz do seu casamento, tanto em relação à atração física e sexual do parceiro, quanto da afinidade do casal e de seus interesses em comum.

De modo oposto, os altos níveis de AN estão associados a baixos índices de satisfação com a vida, satisfação sexual e satisfação com o relacionamento como um todo. Há que se considerar que os $\mathrm{AN}$ influenciam mais a insatisfação no que se refere à sexualidade do que os comportamentos, comprovando as pesquisas que apontam que pessoas mais depressivas ou melancólicas tendem a apresentar 
menor frequência de relações sexuais, preferindo o afastamento e o recolhimento a situações que envolvem contato físico direto ou mesmo situações sociais de forte exposição física (Beam et al., 2011; Seligman, 2011; Spanier, 1976; Tay \& Diener, 2011).

$\mathrm{Na}$ base dessa consideração, pode-se pensar que a atividade sexual estaria relacionada a uma disponibilidade para o contato com o outro, o que se alinha às emoções positivas, como a atitude de disponibilidade para com o outro e de extroversão. Isso explicaria por que as pessoas com maior nível de AP e menor nível de AN mostram também maior satisfação em relação à sexualidade vivenciada com o(a) parceiro(a), como foi encontrado na amostra de casados em apreço. Além disso, há que se considerar que pessoas casadas com níveis mais elevados de $\mathrm{AN}$ demandam maior suporte emocional do(a) parceiro(a) (Beam et al., 2011; Van Steenbergen, Kluwer, \& Karney, 2011), o que sugere a necessidade de que tais pessoas estejam engajadas em relacionamentos mais protetivos e também mais satisfatórios. Pelos dados obtidos no presente estudo, quanto mais elevado o nível de $\mathrm{AN}$, menor a satisfação e, consequentemente, menor a possibilidade de oferta de apoio emocional.

Há que se destacar que a SR não está associada à $\mathrm{SV}$, de modo que os participantes que mostraram estar satisfeitos com o casamento não referiram satisfação em relação à própria vida. Há que se considerar que o BES é uma avaliação acerca de aspectos subjetivos do sujeito, e não objetivos, de modo que os níveis de SV estão relacionados às experiências cotidianas dessas pessoas casadas, e não a aspectos físicos ou materiais que porventura possam ser acrescentados no casamento, como bens materiais, alteração de status econômico, mudança de cidade, entre outros. Esperava-se que a SR ocupasse um papel importante na atribuição da SV, conforme discutido em estudo anterior, que utilizou outras escalas e que comprovou a associação entre satisfação com a vida e satisfação diádica (Scorsolini-Comin \& Santos, 2010). A SV é uma espécie de "balanço" acerca das experiências cotidianas, tendo como base o ideal de satisfação apregoado pelo próprio sujeito. Desse modo, a SV surge da comparação subjetiva com a SV considerada ideal, ou seja, a partir de uma comparação com o que se veicula na sociedade e que se oferece como padrão normativo. Similarmente, quando se questiona para uma pessoa casada se o seu relacionamento é satisfatório, ela evoca não apenas os eventos ocorridos em seu casamento, como os compara com os demais relacionamentos conhecidos ou mesmo idealizados.
A SR também está fortemente correlacionada com a SAFS $(r h o=0,58 ; p \leq 0,001)$ e satisfatoriamente com a SAIC ( $r h o=0,30 ; p \leq 0,001)$, comprovando que a medida unidimensional de satisfação no relacionamento amoroso (SR) empregada neste estudo encontrou apoio nos fatores do instrumento EFS-RC. $\mathrm{Na}$ amostra aqui analisada, destaca-se que tanto a satisfação sexual quanto a satisfação em relação aos comportamentos do parceiro são equivalentes ao questionamento acerca da satisfação geral com o relacionamento.

Em pesquisa com 106 pessoas casadas (Scorsolini-Comin \& Santos, 2012), comprovou-se forte correlação entre a coesão diádica (Spanier, 1976) e a satisfação conjugal, bem como forte associação entre satisfação com a vida (SV) e conjugalidade, o que não foi observado na presente amostra. Esse achado é oposto ao relatado em boa parte da literatura científica, que afirma que o bem-estar experienciado na vida de modo geral está relacionado com os sentimentos de prazer, engajamento e satisfação advindos do casamento (Seligman, 2011; Snyder \& Lopez, 2009; Stones, Worobetz, \& Brink, 2011).

Um ponto a ser discutido é que, no presente estudo, houve alta incidência de indivíduos casados que também relataram que estavam muito satisfeitos com seus relacionamentos, com uma média 4 em uma escala que variava de 1 a 5 , sendo 5 a categoria correspondente a "extremamente satisfeito". Desse modo, pode-se considerar que a amostra foi constituída, predominantemente, por pessoas satisfeitas em seus casamentos. Bertoni e Bodenmann (2010) compararam três grupos: casais satisfeitos, insatisfeitos e em terapia de casal, destacando que os casais em processo terapêutico relatavam maiores níveis de insatisfação do que os próprios casais caracterizados como insatisfeitos, demonstrando que a busca pela psicoterapia coincidia com a percepção de insatisfação conjugal.

Embora o critério de seleção para o presente estudo não exigisse que os casais estivessem satisfeitos ou insatisfeitos, mas apenas casados há pelo menos dois anos e que não estivessem em processo de separação, destaca-se que alguns casais contatados e que relatavam "estar em crise" não quiseram participar da pesquisa. Uma hipótese seria a de que as pessoas engajadas em relacionamentos insatisfatórios teriam pouca disponibilidade e interesse em contribuir com um estudo que destacaria exatamente esse aspecto de suas vidas, ou mesmo que já tivessem amadurecendo uma possível separação. De modo inverso, pessoas envolvidas em relacionamentos satisfatórios teriam maior abertura e interesse em compartilhar suas experiências positivas. 
Provavelmente, a própria "escolha" dos participantes "filtrou" aqueles mais satisfeitos e, possivelmente, com maiores níveis de bem-estar, constituindo um dos vieses que poderiam ser corrigidos em estudos futuros. Posto isso, consideramos a possibilidade de que, em novos estudos, sejam contemplados grupos de pessoas com diferentes níveis de satisfação com o relacionamento, a exemplo do estudo de Bertoni e Bodenmann (2010), embora as estratégias para tanto ainda tenham que ser melhor delineadas. Obviamente, deve-se rever a estratégia de recrutamento dos participantes, diversificando-os de modo a se assegurar a maior variedade possível, talvez, até mesmo, incluindo pessoas em processo de separação. Outra limitação na composição da amostra foi o fato de a maioria dos participantes ser do sexo feminino, o que pode ter influenciado alguns resultados obtidos.

Mas voltemos à hipótese inicial do estudo, de que a conjugalidade dos pais estaria associada à conjugalidade dos filhos. Amparados na literatura psicanalítica, considera-se que os relacionamentos amorosos dos filhos possibilitariam ao respondente uma nova leitura do casamento dos seus pais, o que desloca o eixo de discussão para uma dimensão pessoal, não determinista, e sim relacional, ou seja, a satisfação individual estaria associada a uma possível remalhagem do vínculo filiativo (Benghozi, 2010), notadamente, nos casos em que essas vinculações do casal parental não tenham sido estabelecidas de modo sadio. De modo similar, os laços filiativos do casamento dos filhos também poderiam remalhar os laços filiativos, herdados. A influência é recíproca e segue, portanto, uma via de mão dupla.

Esse posicionamento nos remete ao paradoxo fusão-separação destacado por Magalhães (2009). Por meio dele, compreende-se que, para a estruturação da conjugalidade, os parceiros precisam se separar e se diferenciar de modo adequado de suas famílias de origem, ou seja, abrir espaço para a transformação do vínculo inicial para a constituição da vinculação conjugal. A conjugalidade estaria permanentemente atravessada por esse paradoxo, de modo que não se poderia obter uma fusão total e definitiva, bem como não se chegaria a uma condição de total separação da família de origem. As lembranças infantis acerca da família de origem e seu funcionamento são consideradas fundamentais no sentido de criar uma imagem que represente o conceito de família e de pertencimento a uma estrutura (Bandura, Caprara, Barbaranelli, Regalia, \& Scabini, 2011; Willoughby et al., 2012). Assim, os modelos familiares herdados estariam presentes na configuração do novo casal, ainda que os cônjuges os rejeitassem conscientemente e os escamoteassem (Benghozi, 2010).

Os modelos familiares são recortes submetidos a uma constante atualização, o que possibilita considerar o processo de remalhagem das vinculações ocorrido ao longo do tempo (Benghozi, 2010), permitindo mudanças no modo de se perceber e avaliar o casal parental. De acordo com os dados deste estudo, não é possível afirmar inequivocamente que a percepção sobre a conjugalidade dos pais está correlacionada com a satisfação nos relacionamentos amorosos experienciados pelos filhos, haja vista que houve apenas uma fraca associação, ainda que significativa, entre PCP e SAIC. Por esse achado, pode-se compreender que essa percepção do relacionamento parental possa oferecer sim uma base para a satisfação no casamento em termos de comportamentos e interesses, embora não a explique totalmente, muito menos a determine. Assim, aventa-se que o trabalho de remalhagem do vínculo ao longo do ciclo vital, bem como o processo de fusão-separação na constituição da conjugalidade, possam ser explicações para as mudanças observadas. Apreender melhor o modo como essa remalhagem se processa parece ser um dos desafios para as pesquisas nos campos da conjugalidade e da família.

\section{Conclusões}

A avaliação da conjugalidade ainda é um campo em desenvolvimento, de modo que apreender as mudanças ao longo do ciclo vital no que se refere ao domínio diádico parece ser um desafio importante de ser assumido pelos pesquisadores da Psicologia da Família. Assim, investigar as relações entre conjugalidade dos pais e conjugalidade dos filhos não tem permitido conclusões herméticas. Pelo contrário, na base das explicações das relações existentes todos são convidados constantemente a buscar referências na clínica de casal, por exemplo (Benghozi, 2010), ou mesmo em outros conceitos, como o de bem-estar subjetivo, ampliando as possibilidades de compreensão do fenômeno. Assim, a conjugalidade pode ser estudada a partir de modelos que considerem diferentes conceitos que interferem na estruturação da díade, a exemplo da conjugalidade dos pais e do bem-estar, como priorizados no presente estudo. Uma lacuna que se abre, no entanto, é que os processos de mudança observados ao longo do tempo, sumarizados no conceito de remalhagem dos vínculos, ainda necessitam de mais investigação. 
A afetividade positiva e a maior abertura em relação à vida podem ser fatores a serem considerados no engendramento de novas configurações de família e de conjugalidade, o que é uma das considerações mais fortemente apregoadas pela Psicologia Positiva. Embora este não tenha sido o marco teórico priorizado neste estudo, não podemos desconsiderar a tese de Seligman (2011), que afirma que os relacionamentos interpessoais satisfatórios são fundamentais para conformar a experiência de bem-estar e de satisfação com a vida. Ao final desse percurso, acrescenta-se que a apreciação que o indivíduo faz da conjugalidade comporta também uma dimensão relacional, à medida em que o julgamento que se faz do casamento de outrem passa, inequivocamente, por uma reflexão pessoal e intransferível acerca dos próprios sentimentos e dos relacionamentos amorosos vivenciados, que estabelecem uma base que não é dada a priori e, muito menos, imutável.

Pensando nesse novo paradigma de bem-estar, pessoas envolvidas em relacionamentos considerados "estáveis" (sem qualquer pressuposição de fidelidade ao princípio monogâmico, mas sim de estabilidade emocional ou com certa permanência ao longo do tempo) teriam maior probabilidade de se mostrarem engajadas nessas relações, podendo expressar emoções positivas (contentamento, prazer, alegria, esperança, otimismo), realizarem-se pessoalmente e desenvolverem um sentimento de que a vida vale a pena ser vivida - ou, em outras palavras, de que existe um sentido maior na experiência de viver e se relacionar amorosamente. Essa satisfação experienciada poderia contribuir para remalhar vinculações emocionalmente disfuncionais, possibilitando releituras mais positivas e significativas no domínio familiar, o que ainda deve ser suportado por outros estudos.

\section{Referências}

Ajzen, I., \& Fishbein, M. (1998). Understanding attitudes and predicting social behavior. New Jersey: Prentice-Hall.

Albuquerque, A. S., \& Tróccoli, B. T. (2004). Desenvolvimento de uma escala de bem-estar subjetivo. Psicologia: Teoria e Pesquisa, 20(2), 153-164. Recuperado de http://www.scielo.br/pdf/ptp/v20n2/ a08v20n2

Associação Brasileira dos Institutos de Pesquisa de Mercado [Abipeme] (2008). Critério de classificação socioeconômica - Brasil. São Paulo, SP: Autor.
Bandura, A., Caprara, G. V., Barbaranelli, C., Regalia, C., \& Scabini, E. (2011). Impact of family efficacy beliefs on quality of family functioning and satisfaction with family life. Applied Psychology, 60, 337-518. doi: 10.1111/j.1464-0597.2010.00442.x

Beam, C. R., Horn, E. E., Hunt, S. K., Emery, R. E., \& Turkheimer, E. (2011). Revisiting the effect of marital support on depressive symptoms in mothers and fathers: A genetically informed study. Journal of Family Psychology, 25, 336-344. doi: $10.1037 / \mathrm{a} 0023758$.

Benghozi, P. (2010). Malhagem, filiação e afiliação - Psicanálise dos vínculos: Casal, família, grupo, instituição e campo social (E. D. Galery, Trad.). São Paulo: Vetor.

Bertoni, A., \& Bodenmann, G. (2010). Satisfied and dissatisfied couples positive and negative dimensions, conflict styles, and relationships with family of origin. European Psychologist, 15, 175-184. doi: 10.1027/1016-9040/a000015

Bowlby, J. (1990). Apego e perda: Apego - A natureza do vínculo (A. Cabral, Trad.). São Paulo: Martins Fontes. (Original publicado em 1969).

David, D. H., Gelberg, L., \& Suchman, N. E. (2012). Implications of homelessness for parenting young children: A preliminary review from a developmental attachment perspective. Infant Mental Health Journal, 33, 1-9. doi: 10.1002/imhj.20333

Féres-Carneiro, T., Ziviani, C., \& Magalhães, A. S. (2007). Questionário sobre a conjugalidade dos pais como instrumento de avaliação. In T. Féres-Carneiro (Ed.), Família e casal: Saúde, trabalho e modos de vinculação (pp. 251-268). São Paulo: Casa do Psicólogo.

Hare, A. L., Miga, E. M., \& Allen, J. P. (2009). Intergenerational transmission of aggression in romantic relationships: The moderating role of attachment security. Journal of Family Psychology, 23, 808-818. doi: $10.1037 / \mathrm{a} 0016740$.

Instituto Brasileiro de Geografia e Estatística [IBGE] (2011). Estatísticas do Registro Civil de 2010. Rio de Janeiro: Autor. Recuperado de http://www.ibge. gov.br/home/estatistica/populacao/registrocivil/2010/rc2010.pdf

Krejcie, R. V., \& Morgan, D. W. (1970). Determining sample size for research activities. Educational and Psychological Measurement, 30, 
607-610. Recuperado de http://www.researchgate.net/publictopics.PublicPostFileLoader.htm $1 ? \mathrm{id}=5595878060614 \mathrm{~b} 65 \mathrm{~b} 58 \mathrm{~b} 45 \mathrm{f} 2 \& \mathrm{key}=1321$ 6a60-a154-4314-802d-c4c238788114

Lau, S. R., Beilby, J. M., Byrnes, M. L., \& Hennessey, N. W. (2012). Parenting styles and attachment in school-aged children who stutter. Journal of Communication Disorders, 45, 98-110. doi:10.1016/j. jcomdis.2011.12.002

Magalhães, A. S. (2009). Conjugalidade e parentalidade na clínica com famílias. In T. Féres-Carneiro (Ed.), Casal e família: Permanências e rupturas (pp. 205-217). São Paulo: Casa do Psicólogo.

Marin, A. H., Piccinini, C. A., \& Tudge, J. R. H. (2011). Estabilidade e mudança nas práticas educativas maternas e paternas ao longo dos anos pré-escolares da criança. Psicologia: Reflexão e Crítica, 24, 71-79. doi: 10.1590/S0102-79722011000100009

Moraes, S. A., \& Souza, J. M. P. (1998). Metodologia caso-controle em epidemiologia de doenças cardiovasculares. Revista de Saúde Pública, 32, 82-88. Recuperado de http://www.revistas.usp.br/rsp/ article/view/24349/26273

Moreira, L. V. C., Rabinovich, E. P., \& Silva, C. N. (2009). Olhares de crianças baianas sobre família. Paidéia (Ribeirão Preto), 19(42), 77-85. Recuperado de http://www.redalyc.org/articulo. oa?id $=305423764010$

Pasquali, L. (Org.). (2001). Técnicas de exame psicológico: Manual. São Paulo: Casa do Psicólogo.

Scorsolini-Comin, F., \& Santos, M. A. (2010). Satisfação com a vida e satisfação diádica: Correlações entre construtos de bem-estar. PsicoUSF, 15, 249-256. doi: 10.1590/S1413-82712010000200012

Scorsolini-Comin, F., \& Santos, M. A. (2012). Correlations between subjective well-being, dyadic adjustment and marital satisfaction in Brazilian married people. The Spanish Journal of Psychology, 15, 166-176. Recuperado de http://www.ncbi.nlm. nih.gov/pubmed/22379707

Seligman, M. E. P. (2011). Florescer: Uma nova compreensão sobre a natureza da felicidade e do bem-estar (C. P. Lopes, Trad.). Rio de Janeiro: Objetiva.

Snyder, C. R., \& Lopez, S. J. (2009). Psicologia Positiva: Uma abordagem científica e prática das qualidades humanas. (R. C. Costa, Trad.). São Paulo: Artmed.
Spanier, G. B. (1976). Measuring dyadic adjustment: New scales for assessing the quality of marriage and similar dyads. Journal of Marriage and the Family, 38, 15-28. doi: 10.2307/350547

Stones, M. J., Worobetz, S., \& Brink, P. (2011). Overestimated relationships with subjective well-being. Canadian Psychology, 52, 93-100. Recuperado de http://psycnet.apa.org/index.cfm?fa=buy. optionToBuy\&id=2011-10782-003

Tay, L., \& Diener, E. (2011). Needs and subjective well-being around the world. Journal of Personality and Social Psychology, 101, 354-365. doi: 10.1037/ a0023779

Van Steenbergen, E. F., Kluwer, E. S., \& Karney, B. R. (2011). Workload and the trajectory of marital satisfaction in newlyweds: Job satisfaction, gender, and parental status as moderators. Journal of Family Psychology, 25, 345-355. doi: 10.1037/a0023653

Vieira, J. M., Ávila, M., \& Matos, P. M. (2012). Attachment and parenting: The mediating role of work-family balance in Portuguese parents of preschool children. Family Relations, 61, 31-50. doi: 10.1111/j.1741-3729.2011.00680.

Wachelke, J. F. R., Andrade, A. L., Souza, A. M., \& Cruz, R. M. (2007). Estudo complementar da validade fatorial da escala fatorial de satisfação em relacionamento e predição de satisfação global com a relação. PsicoUSF, 12, 221-225. Recuperado de http://www.scielo.br/pdf/pusf/v12n2/ v12n2a10.pdf

Willoughby, B. J., Carroll, J. S., Vitas, J. M., \& Hill, L. M. (2012). "When are you getting married?": The intergenerational transmission of attitudes regarding marital timing and marital importance. Journal of Family Issues, 33, 223-245. doi: 10.1177/0192513X11408695

Ziviani, C., Féres-Carneiro, T., \& Magalhães, A. S. (2011). Sons and daughters' perception of parents as a couple: Distinguishing characteristics of a measurement model. Psicologia: Reflexão e Crítica, 24, 28-39. doi: 10.1590/S0102-79722011000100005

Recebido: 25/11/2014

Primeira reformulação: 11/02/2015 Aprovado: 18/02/2015 
Nota dos autores:

Este artigo é derivado da tese de doutorado do primeiro autor, defendida junto ao Programa de Pós-Graduação em Psicologia da Faculdade de Filosofia, Ciências e Letras de Ribeirão Preto da Universidade de São Paulo. Este estudo foi subvencionado pelo Programa Santander de Bolsas de Mobilidade Internacional, pela Coordenação de Aperfeiçoamento de Pessoal de Nível Superior (CAPES) e pela Fundação de Amparo à Pesquisa do Estado de São Paulo (Processo FAPESP 2010/00244-9).

Sobre os autores:

Fabio Scorsolini-Comin é doutor em Psicologia pela Faculdade de Filosofia, Ciências e Letras de Ribeirão Preto da Universidade de São Paulo (FFCLRP-USP), com pós-doutorado em Tratamento e Prevenção Psicológica pela mesma instituição (Bolsa de Pós-Doutorado Júnior do CNPq). Professor adjunto do Departamento de Psicologia da Universidade Federal do Triângulo Mineiro (UFTM).

E-mail: fabioscorsolini@gmail.com

Anne Marie Germaine Victorine Fontaine é professora catedrática da Faculdade de Psicologia e Ciências da Educação da Universidade do Porto (FPCE-UP), Porto, Portugal.

E-mail: fontaine@fpce.up.pt

Sabrina Martins Barroso é doutora em Saúde Pública pela Universidade Federal de Minas Gerais (UFMG) e professora adjunta do Departamento de Psicologia da Universidade Federal do Triângulo Mineiro (UFTM), Uberaba, MG - Brasil.

E-mail:smb.uftm@gmail.com

Manoel Antônio dos Santos é doutor em Psicologia Clínica pelo Instituto de Psicologia da Universidade de São Paulo (IP-USP) e livre-docente pela Faculdade de Filosofia, Ciências e Letras de Ribeirão Preto da Universidade de São Paulo (FFCLRP-USP), Ribeirão Preto, SP - Brasil. É Professor Associado 3 do Departamento de Psicologia da FFCLRP-USP e bolsista de Produtividade em Pesquisa do CNPq nível 1B.

E-mail: masantos@ffclrp.usp.br

Contato com os autores:

Prof. Dr. Fabio Scorsolini-Comin

E-mail: fabioscorsolini@gmail.com

Departamento de Psicologia da Universidade Federal do Triângulo Mineiro (UFTM)

Av. Getúlio Guaritá, 159, $3^{\circ}$ andar, Abadia, Uberaba/MG - Brasil.

CEP: $38025-440$ 